\title{
High-Q microsphere cavity for laser stabilization and optoelectronic microwave oscillator
}

\author{
Vladimir S. Ilchenko", X. Steve Yao, Lute Maleki \\ Jet Propulsion Laboratory, California Institute of Technology, \\ 4800 Oak Grove Drive, Pasadena, CA $91109-8099$
}

\begin{abstract}
With submillimeter size and optical $Q$ up to $\sim 10^{10}$, microspheres with whispering-gallery (WG) modes are attractive new component for fiber-optics/photonics applications and a potential core in ultra-compact high-spectral-purity optical and microwave oscillators. In addition to earlier demonstrated optical locking of diode laser to WG mode in a microsphere, we report on microsphere application in the microwave optoelectronic oscillator, OEO. In OEO, a steady-state microwave modulation of optical carrier is obtained in a closed loop including electro-optical modulator, fiber-optic delay, detector and microwave amplifier. OEO demonstrates exceptionally low phase noise $(-140 \mathrm{dBc} / \mathrm{Hz}$ at $10 \mathrm{kHz}$ from $\sim 10 \mathrm{GHz}$ carrier) with a fiber length $\sim 2 \mathrm{~km}$. Current technology allows to put all parts of the OEO, except the fiber, on the same chip. Microspheres, with their demonstrated $Q$ equivalent to a kilometer fiber storage, can replace fiber delays in a truly integrated device. We have obtained microwave oscillation in microsphere-based $O E O$ at 5 to $18 \mathrm{GHz}$, with 1310nm and 1550nm optical carrier, in two configurations: 1)with external DFB pump laser, and 2)with a ring laser including microsphere and a fiber optic amplifier. Also reported is a simple and efficient fiber coupler for microspheres facilitating their integration with existing fiber optics devices.
\end{abstract}

Keywords: Microspheres, fiber optics, microwave photonics

\section{INTRODUCTION}

High-Q optical microsphere resonators with whispering-gallery (WG) modes [1-3], thereafter microspheres, have attracted growing interest in experimental cavity-QED [4-6], microlasers [7-9], measurement science and other photonics applications $[10,11]$. Following extensive research of Mie resonances in microdroplets of aerosols [12], current studies focus mostly on the properties and applications of laboratory-fabricated solid-state microspheres with highly confined WG modes. For diameter of the sphere exceeding a few tens of free-space wavelengths, curvature-related losses become negligible, resulting in radiative quality-factors $Q_{\mathrm{rad}} \sim 10^{20}$ and larger. Scattering losses on residual surface inhomogeneities can also be made small enough so that the quality-factor as high as $Q \sim 10^{10}$, limited only by intrinsic attenuation, can be obtained in high-purity fused silica microspheres in the visible and near-infrared band $[13,14]$. Typical diameter of the spheres is in the order of hundreds of microns. Based on this, very high quality-factor $Q \geq 10^{11}$ can be expected in the fiber optics comminication band $1.3-1.55 \mu \mathrm{m}$ that coincides with the minimum of attenuation in silica waveguides.

The unique combination of high $Q$ and submillimeter dimensions makes microspheres an attractive and novel building block for fiber optics and photonics devices. Aside from the obvious apllication such as in compact narrow-band optical filters and spectrum analyzers. microspheres can be a basis for variety of sensors that employ the sensitivity of whispering-gallery mode frequencies and $Q$ to variations of temperature. applied stress. or parameters of the ambient medium, which is exposed to the modes' evanescent field.

In this paper, we focus on the microsphere application for the realization of compact high-coherence oscillators in optical and microwave band. As shown earlier [ 15$]$. the intrinsic feedback mechanism in microspheres (intracavity backscattering) can be used for simple optical locking of the diode lasers. and the possibility of creation of ultra-compact sub-kHz linewidth laser source is already demonstrated. High-Q microspheres can be as well a basis for a very small-size high-spectml purity photonic microwave source as they replace kiloncter-long fiber-optic delays in the Opto-Electronic Oscillator [16] (OEO).

- Correspondence Email: ilchenkoighorology.jpl nilsil.gov: Telephone: $81835+8485$ : Fax: 8183936773 


\section{THE QUALITY-FACTOR AND SPECTRAL PROPERTIES}

High quality-factor is the basis for the interest in most of the suggested applications of microspheres. since it is the parameter which defines the resolution in the spectroscopy. filtering and sensors, and is capable of lowering the threshold in microlasers and suppression of phase and frequency noises in microsphere-based oscillators. Microspheres as dielectric cavities are compact alternatives to high-finesse Fabry-Perot (FP) optical cavities comprising of two high-reflectivity mirrors. In contrast to FP cavities where the $Q$ scales down proportionally with the decreasing mirror separation. microspheres have their $Q$ ultimately limited by the fundamental attenuation in the material, as other mechanisms of dissipation can be eliminated in the spheres of submillimeter size. In FP cavities, even if the separation between ultra-high finesse mirrors is small, they have to remain relatively large to prevent the increase of diffraction losses.

As earlier reported, quality-factor of WG modes is determined by several loss factors:

$$
Q^{-1}=Q_{\text {rad }}^{-1}+Q_{\text {s.s. }}^{-1}+Q_{\text {cont }}^{-1}+Q_{\text {mat }}^{-1}
$$

where $Q_{\text {rad }}^{-1}$ denotes intrinsic radiative losses; $Q_{s . s}^{-1}$ - scattering losses on residual surface inhomogeneities; $Q_{\text {cont }}^{-1}$ - losses introduced by surface contaminants, and $Q_{\text {mat }}^{-1}$ - material losses.

$Q_{\text {rad }}^{-1}$ vanishes exponentially with increasing size so that it can be neglected for $D / \lambda \geq 50$ ( $D$ is microsphere diameter, $\lambda=$ wavelength). $Q_{\text {s.s. }}^{-1}$ can be dominant for intermediate-size spheres:

$$
Q_{\text {s.s. }}=\frac{\lambda^{2} D}{2 \pi^{2} \sigma^{2} B} \approx 7.5 \times 10^{9} \frac{D[\mu m]}{100} \text {, }
$$

where $n$ - refraction index; $\sigma$ and $B$ - the rms size and correlation length of surface inhomogeneities. This estimate relies on parameters of silica and the reported $\sigma=0.3 \mathrm{~nm}$ and $B=3 \mathrm{~nm}$ for glass surfaces and indicates that $Q_{s .5}^{-1} \ll 1 \times 10^{-10}$ may be expected only in relatively "large" spheres $D>100 \mu \mathrm{m}$. In the absence of contaminants, therefore, the $Q$ of "large" spheres may reach the limit defined by material losses $Q_{\text {mat }}^{-1}$. Optical attenuation in fused silica, investigated intensely in the context of fiber transmission optimization, at $633 \mathrm{~nm}$ is $\alpha \geq 7 \mathrm{~dB} / \mathrm{km}$ ( $5 \mathrm{~dB}$ is bulk Rayleigh scattering and $2 \mathrm{~dB}$ is absorption). Therefore the principal limit for microsphere $Q$ at $633 \mathrm{~nm}$ is

$$
Q_{\text {mat }}=\frac{2 \pi n}{\alpha \lambda} \leq 0.9 \times 10^{10}
$$

Indeed, in the experiments in the visible and near infrared band, based on the measurement of the ringdown time $\tau$ of free oscillations, $Q=(0.6 \ldots 0.8) \times 10^{10}$ has been obtained in micropsheres of diameter 600 to $800 \mu \mathrm{m}[13,14]$. (The corresponding ringdown (energy storage) times were from about $\tau=3 \ldots+\mu \mathrm{s}$, equivalent to about $0.8 \mathrm{~km}$ long fiber delay.) It was proved. however. that under conditions of normal atmospheric humidity, the quality-factor of the spheres is subject to deterioration within several-minute scale, down to the value of $(1 \ldots 3) \times 10^{9}$. The responsible mechanism was identified as adsorption of a monolayer of atmospheric water, so that preservation of the ultimate $Q$ requires manipulation in dry environment. or fast packaging into sealed devices. Larger $Q$ can be expected closer to minimum of attenuation in fused silica $\alpha=0.2 \mathrm{~dB} / \mathrm{km} ; Q_{\text {mat }}^{-1} \geq 1 \times 10^{11}$ at $\lambda=1.55 \mu \mathrm{m}$. with corresponding energy storage time $\tau \sim 0.1 \mathrm{~ms}$. We are currently conducting experiments to determine whether this high $\mathrm{Q}$ can be realized experimentally. The difficulty evidently is that $\mathrm{OH}$ related optical absorption, responsible for environmental deterioration of microsphere $Q$, has its peaks located near the reported minimum of attenuation in silica. We can also mention here that some of prospective fiber materials, although not ready for fiber drawing, have been predicted to have smaller attenuation than fused silica (sodium-magnesium silicate glass. $\alpha=0.06 \mathrm{~dB} / \mathrm{km}[17])$. and may be suitable for microsphere fabrication. 
It is worth emphasizing here that even easily achievable $Q=10^{8} \ldots 10^{9}$ in silica microspheres is many orders of magnitude higher that the quality-factor of present-day planar microfabricated cavities (rings and disks) [18], which offers a new domain of opportunities for the creation of ultra-compact filters, sensors and high-coherence oscillators.

The spectrum of WG modes in microspheres is significantly more complicated compared to that of FP cavities and singlemode waveguide rings. In ideal spheres, as known from early analyses [19], the spectrum can be described by $T E(T M)_{\text {tma }}$ modes, $m= \pm l, \ldots, 0$, which are degenerate with respect to the index $m$ and form several superimposed quasi-equidistant grids defined by successive integer numbers of wavelengths packed along the circumference of the sphere $\pi n \approx \approx \lambda$ Here $D$ is the diameter of the sphere; $n$ is the refraction index of the material, and $\lambda$ is the free-space wavelength. The "fundamental" free spectral range, associated with successive $l$, is $F S R=0.2 \ldots 1 \mathrm{THz}$ for $D=100-500 \mu \mathrm{m}$. Index $q$ accounts for the number of mode field variations along the radial extent of the mode. Index $m$ defines the number of mode field maxima in "meridional" direction, which is equal to $|1-\mathrm{m}|+1$. The term "whispering-gallery" modes is normally referred to those confined near the surface $q \sim 1$, in a narrow band surrounding the sphere "equator" $(l-m)<<l$.

The real cavities are slightly elliptical, and the ellipticity lifts the degeneracy versus $m$ index. Each of the TE(TM) resonances with given $l, q$ is split into $l+1$, corresponding to individual $|m|$ and separated by "small" FSR $\approx(c / \pi D n)\left(m \varepsilon^{2} / l\right)$; where $\varepsilon$ is the eccentricity of the cavity [20]. The value of small FSR in laboratory made microspheres (formed by fusion of silica fiber preforms) is typically between 1 and $10 \mathrm{GHz}$; for a $\varepsilon^{2} \sim(1 \ldots 3) \times 10^{-2}$, and $D=100-500 \mu \mathrm{m}$

Despite the fact that one might expect a huge number of modes observable in the microsphere spectra, spacial selection provided by mode-matched coupling allows to excite a limited number of modes with selected $q, l$ and polarization, near agiven wavelength [21].

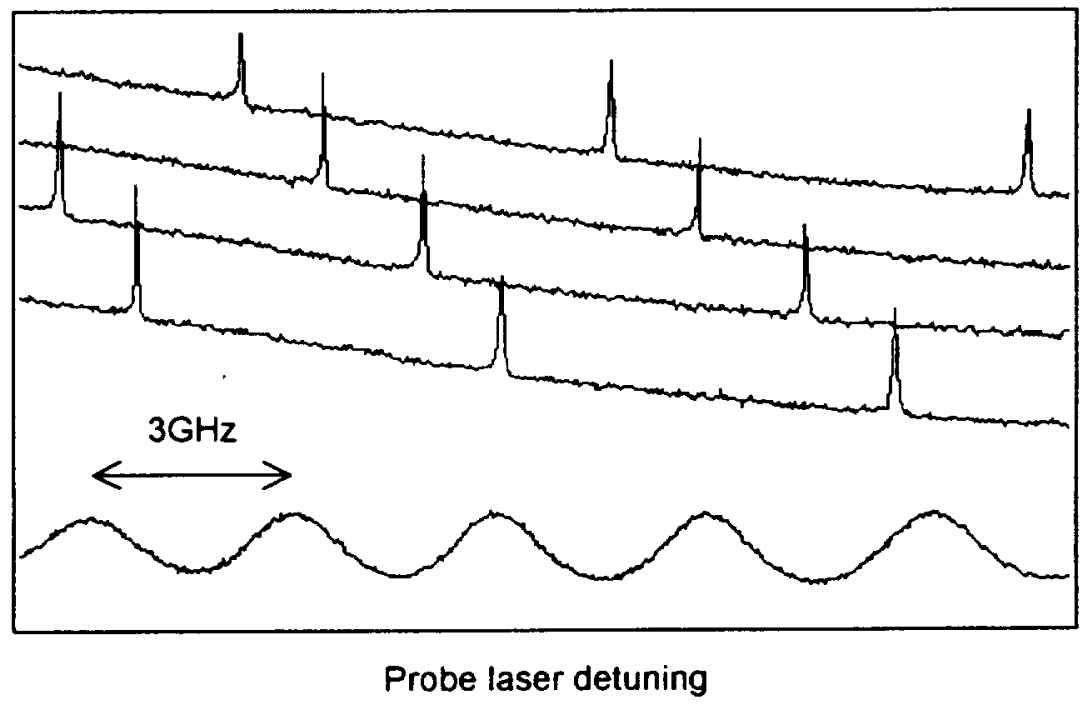

Fig.1. A whispering-gallery mode spectrum of a fused silica microsphere and its modilication upon PZT-assisted axial compression (mechanical tuning) [22]. Diameter $160 \mu \mathrm{m}$, eccentricity $\vec{\varepsilon} \sim 2.6 \times 10^{-2}$, wavelength $\lambda=807 \mathrm{~nm}, 1 \approx 900$; "small" $\mathrm{FSR}=5.3 \mathrm{GHz}$. Lower curve is the tringes of low-linesse calibration FP interterometer. Coupling setup addresses the $\pi I_{L m I}$ modes; $m \approx 1-20$; unloaded $Q$ of the tunable sphere $Q \sim 1 \times 10^{8}$

A typical spectrum of fused silica microsphere cavity observed upon frequency scanning of a probe laser. and its modification upon mechanical tuning via PZT-assisted axial compression of the sphere [22] is given in Fig. I.

Relatively large actual FSR in microspheres within the microwave band. simplifies mode selection in laser locking application of microspheres. and can act as a direct frequency reference for a microsphere-based optoclectronic microwave oscillator. 


\section{NEAR-FIELD COUPLERS: EFFICIENCY AND OPTIMIZATION. A SIMPLE FIBER-OPTIC COUPLER FOR MICROSPHERES}

As already mentioned above. WG modes possess extremely small radiative losses. arc confined to the interior of the microsphere and cannot be excited by free-space beams. If no modification (such as grating) is made on the sphere surface, the coupling has to be provided by the appropriate near-field devices. Presently, in addition to the well-known prism coupler with frustrated total internal reflection $[1-3,21]$, demonstrated coupler devices include sidepolished fiber coupler $[11,23]$ and fiber taper [24]. The principle of all these devices is based on providing efficient energy transfer to the circular total-internalreflection-guided wave in the resonator (representing the WG mode) through the evanescent field of a guided wave or a TIR spot in the coupler. Reasonable coupling efficiency has been demonstrated with three types of devices (up to few tens of percents of input beam energy absorbed in a mode at resonance). A systematic theoretical approach has been recently developed to quantify the performance of coupler devices for WG modes [25]. Efficient coupling maybe expected upon fulfillment of two main conditions: 1)mode matching and 2)sufficient coupling strength to provide the buildup of a WG mode with a given intrinsic losses. The first condition can be expressed as follows:

$$
\Gamma \equiv\left(T \cdot B^{i n} / T B^{i n}\right) \rightarrow 1
$$

Here $\Gamma$ is the mode matching coefficient; vector $T / T$ describes the normalized distribution of modes which are excited by the microsphere in the coupler, and $B^{\text {in }} / B^{\text {in }}$ describes the modal content of the input wave propagating in the coupler. Both $T / T$ and $B^{\text {in }} / B^{\text {in }}$ may contain guided modes of the coupler device, or free-space modes, such as focused Gaussian beams in the prism, and also idle (leaky or cladding) modes in the fiber.

The second condition of efficient coupling is best quantified by introducing the coupling quality-factor $Q_{v}$ to account for the additional energy escape from the microsphere mode through the coupler. If the coupling efficiency is characterized by the fractional depth $K^{2}$ of the resonance dip in the transmitted intensity observed upon varying the frequency of the exciting wave in the coupler, then it can be expressed in the following way through $Q_{c}$ and the intrinsic quality-factor of the mode $Q_{o}$ :

$K^{2}=+Q_{o} Q_{c} \Gamma^{2} /\left(Q_{0}+Q_{c}\right)^{2}$

The resonance width of the observed resonance will be defined by the loaded quality-factor $Q_{L}=\left(Q_{0}{ }^{-1}+Q_{c}{ }^{-1}\right)^{-1}$.

With complete mode matching $\Gamma=1$, the critical coupling $K^{2}=1$ (or $100 \%$ absorption of the input wave in resonance) is possible when $Q_{c}$ can be made equal or smaller than $Q_{0}$. Near-exponential decay of the evanescent wave makes the coupling losses a function of the gap $d$ between the sphere and the coupler. If $Q_{\sigma}(0)$ is smaller than $Q_{a}$, one can always decrease the coupling by increasing the gap, to achieve critical coupling at $Q_{c}(d)=Q_{o}$.

(a)

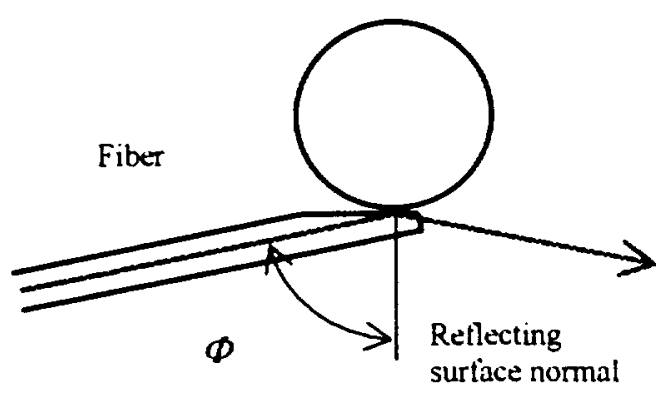

(b)

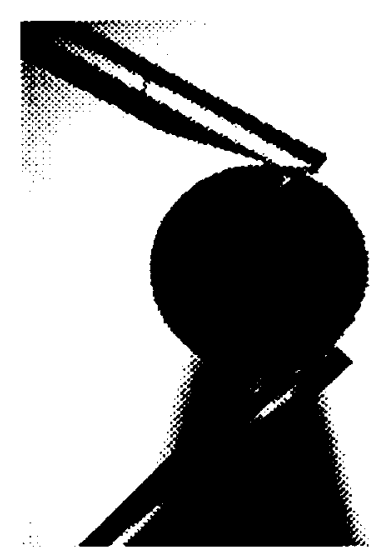

Fig 2. A simple direct tiber coupler lor WG inodes in mucrosplkeres a sichematic; bxilose-up of a silica microsphere (diameter $f 70 \mu \mathrm{m}$ ) with two angle-polished liber couplers 
Prospects for microsphere applications have suffered from the lack of simple and compact coupler devices compatible with modem fiber-optic hardware. Although proved to be most flevible and versatile. the prism coupler is bulky and requires collimation and focusing optics to work with optical fiber. The previously reported attempts to directly couple a microsphere to an optical fiber had limited efficiency due to rernaining phase mismatch (side-polished bent fiber coupler [23]), or still considerable size including few-cm core-to-cladding transformers (tapered monomode fiber coupler [24]). Here. we report a new and simple method of direct fiber coupling to high-Q WG modes in microsphere, which in essense is a hybrid of waveguide and prism coupler.

Schematic of the new coupler and a close-up of an actual setup with microsphere and two couplers is shown in Fig. 2.

The tip of a cleaved single-mode fiber is angle-polished under steep angle. Upon incidence on the angled surface, the light propagating inside the fiber core undergoes total internal reflection and escapes the fiber. With the sphere positioned in the range of the evanescent field from the core area, the configuration provides efficient energy exchange in resonance between the waveguide mode of single-mode fiber and the whispering-gallery mode in the sphere. The angle of the polish is chosen to secure the phase matching requirement: $\Phi=\arcsin \left(n_{\text {spher }} / n_{f b e r}\right)$. Here $n_{\text {fber }}$ stands for the effective refraction index to describe the guided wave in the fiber core truncation area, and $n_{\text {sphere }}$ stands for the effective refraction index to describe azimuthal propagation of WG modes (considered as closed waves undergoing total internal reflection in the sphere). Since the linear dimensions of the angle-cut core area match well the area of evanescent field overlap, the new system is equivalent to a prism coupler with eliminated collimation/focusing optics. The effective refraction index to describe the azimuthal propagation of WG modes near the surface of the sphere can be calculated, for example, on the basis of asympthotic expressions [26] for WG mode frequencies $\omega_{l_{q}}$, where $n_{\text {sphere }}=2 c l / D \omega_{r_{q}}$.

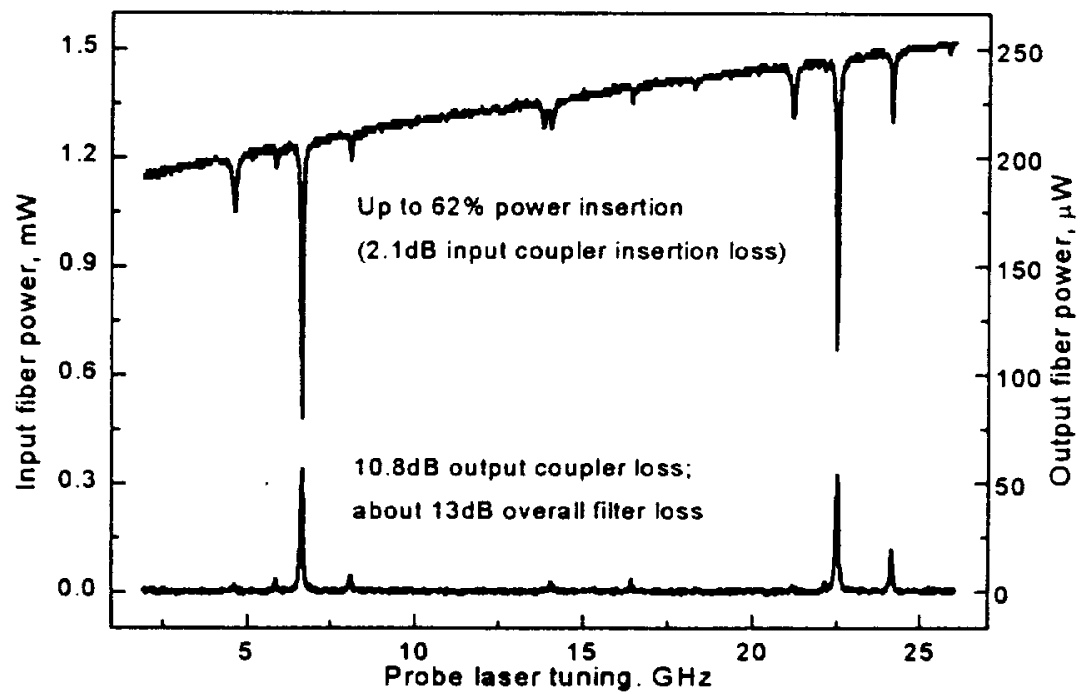

Fig.3. Characterization of the coupling efficiency of angle-polished tiber coupler assembly with fused silica microsphete The slope of the upper curve is due to variation of the probe $1310 \mathrm{~nm}$ DFB laser power upon tuning

For the microsphere with diameter $D=470 \mathrm{~mm}$ at the wavelength $\lambda=1310 \mathrm{~nm}$ (effective index $n_{\text {sphere }}=1 .+26$ for $T E_{l m l}$ modes), and the SMF-28 fiber we used $\left(n_{\text {fiber }}=1.4625\right)$, the optimal angle was $\Phi=77.6^{\circ}$. Presented in Fig. 3 are the experimental results characterizing the performance of the new coupler (unloaded quality-factor of the sphere in this experiment was $Q_{o}=1.1 \times 10^{8}$ ). The two curves present 1)the intensity of the wave escaping the input coupler tip and 2)intensity of the light fed through into the output coupler. upon frequency tuning of the probe DFB laser.

As seen in Fig.3. efficiency of the new angle-polished fiber coupler (over $60 \%$ power insertion in the mode) is comparable to the best previously reported coupler devices (prism and fiber taper). Relativly poor (about $10 \%$ ) outcoupling efficiency in our first experiment was due to insufficient flexibility of the translation stage that provided the coplanar alignment of the two couplers with respect to the symmetry plane of residual non-sphericity of the cavity. It is evident, however. Ihat with improved alignument. efficiency of the coupling can be symmetrized, resulting in overall $\sim \mathrm{dB}$ insertion loss in the system 
considered as narrow-band transmission filter. Further optimization of the coupling (to approach OdB losscs - critical coupling - at resonance) may require additional optimization of the index and/or cross section of the fiber core.

\section{OPTICAL LOCKING OF DIODE LASERS}

Intrinsic optical feedback mechanism in microspheres (intracavity backscattering) makes possible a simple method [27] for optical freqiency locking in semiconductor lasers. As soon as a mode-matching input wave is launched into the coupler device, it excites a circular wave corresponding to one of two possible directions of light circulation in the microsphere modes. Due to the predominant residual scattering of light into oppositely-directed WG mode of the same frequency and configuration. if the quality-factor of the sphere is high enough, an intense counter-propagating wave is excited in the coupler

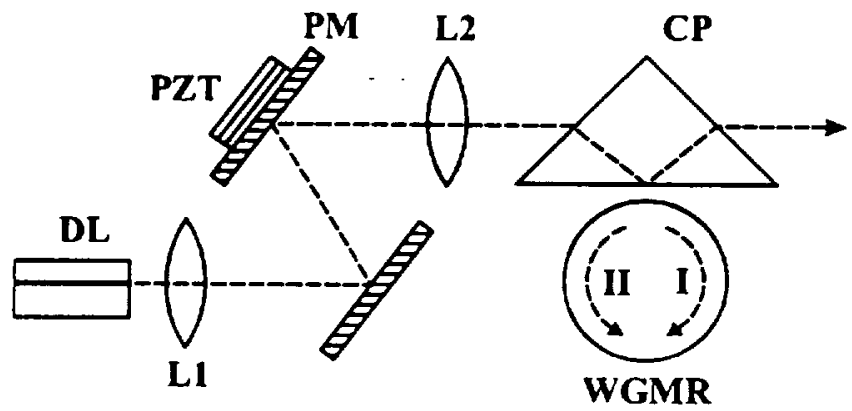

(a)

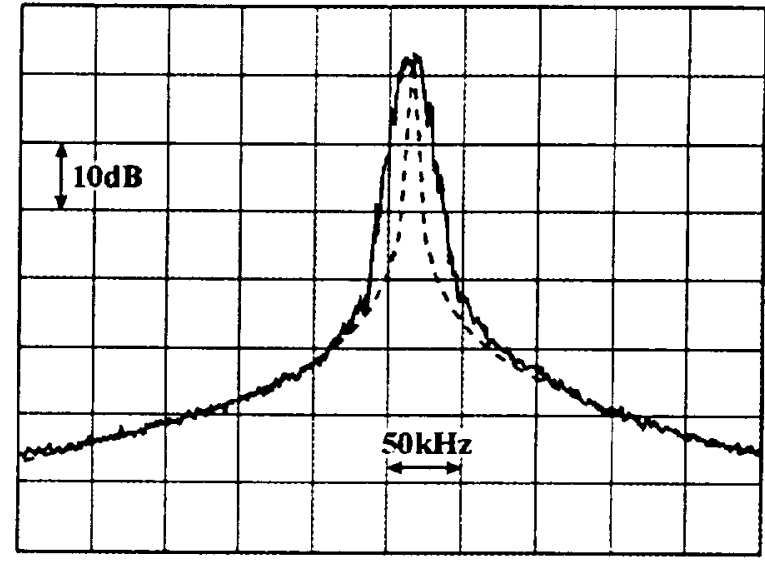

(b)
Frequency

Fig.4. Frequency locking of diode laser to high-Q whispering-gallery microsphere resonator (WGMR): a)schematic of the setup; b)power spectrum of $607 \mathrm{MHz}$ beatnote of two lasers locked to orthogonal-polarization modes in a microsphere. Free-running laser linewidth $\sim 15 \mathrm{MHz}$, loaded Q of the sphere $5 \times 10^{7}$; dashed line is the lorentzian tit with natural linewidth parameter $720 \mathrm{~Hz}$ [27].

and is sent towards the laser. This optical feedback can be easily adjusted by varying the gap between the sphere and the coupler, and easily causes the frequency pulling effect, which locks the laser frequency at the resonance of the microsphere mode. In a tabletop experiment, the dramatic decrease of laser linewidth is demonstarted from about $15 \mathrm{MHz}$ in free-running laser, $\lambda=852 \mathrm{~nm}$, to under $100 \mathrm{kHz}$, upon frequency locking to a microsphere with the loaded quality-factor $Q=5 \times 10^{7}$ (diameter $D=350 \mu \mathrm{m}$ ). The laser was coupled to a WG mode in a microsphere using separate collimator lens, focusing lens and a prism for microsphere coupling. The phase of the optical feedback was stabilized by PZT-assisted adjustment of the free-beam path. With two separate diode lasers indpendently locked to orthogonal polarization WG modes in the same microsphere, the beatnote signal was obtained at $-600 \mathrm{MHz}$ and analyzed using the $\mathrm{RF}$ specrum analyzer. The beatnote spectrum (Fig.4) contains about $20 \mathrm{kHz}$-wide low-frequency part (originated from phase noise due to incompletely suppressed free-path fluctuations) and high-frequency wings fitting the lorentzian profile with $720 \mathrm{~Hz}$ natural linewidth.

(a)

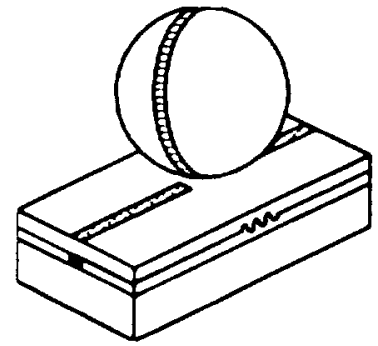

(b)

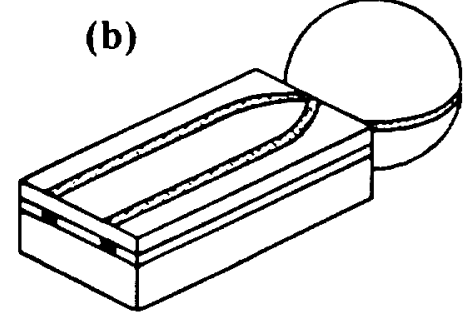

Fig.j. Possible conligurations of compact high-coherence diode lasers using dircet laset chip coupling to a microsphere 
In a simpler configuration, the microsphere can be brought immediately next to the laser chip. allowing a very compact and high-coherence optical oscillator as soon as the modified geometry of the laser chip enibles efficient direct coupling into the WG modes. Some possible configurations are presented in Fig. 5 (reproduced from [27]). Direct coupling from the laser diode into the sphere can be obtained by using a grating imposed on the chip (Fig.5a). V-shaped laser geometry with total intemal reflection at the comer (Fig.5b), or simply by angle-cleaving the chip under the angle of synchronism.

\section{OPTOELECTRONIC MICROWAVE OSCILLATOR}

The Opto-Electronic Oscillator (OEO) [16] is a novel, high-spectral purity source generating an electrical reference signal at microwave frequency, which is also available as the modulation of the laser light carrier. The oscillation begins in a closed loop of positive feedback formed by an electro-optic modulator, a fiber delay. a high-speed photodetector, a microwave filter and an amplifier. The operating frequency is set at one of the integers of total inverse delay time in the feedback loop: the phase noise is highly suppressed with the increasing energy storage time of the delay, thus requiring long fibers. The OEO exhibits very small phase noise $-140 \mathrm{dBc} / \mathrm{Hz}$ at $10 \mathrm{kHz}$ from $10 \mathrm{GHz}$ carrier, with $2 \mathrm{~km}$-long fiber delays and presents a competitive alternative to crystal-based synthesizers and dielectric-resonator-based oscillators. It also possesses a unique property of a direct photonic output, which is important for stable frequency- and time signal distribution and communication applications. All components of the OEO, except the fiber delay, can be produced by microfabrication technologies and may be combined on a single chip.

Microsphere cavities, with their photon storage times in a few-microsecond range (equivalent to about one kilometer propagation length), can serve as a replacement for bulky spools of fiber, opening the path to a truly compact hybrid OEO: Since the mode spacing in microspheres ("small" free spectral range) falls directly into the microwave band, the microspherebased OEO does not require a narrow-band microwave filter and can oscillate at a frequency offset between two modes.

In our preliminary experiments, we have obtained microwave oscillation in the microsphere-based OEO, in two configurations: 1)with external DFB pump laser and 2)with the ring laser including the microsphere and the optical amplifier. The schematic of the two configurations is presented in Fig. 6.

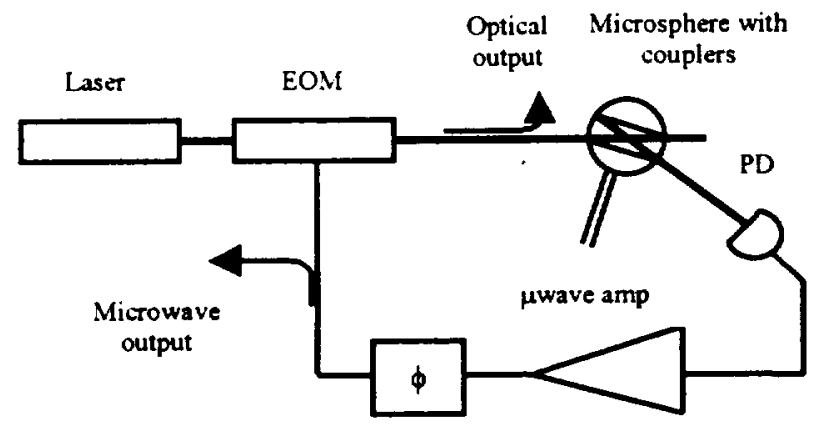

(a)

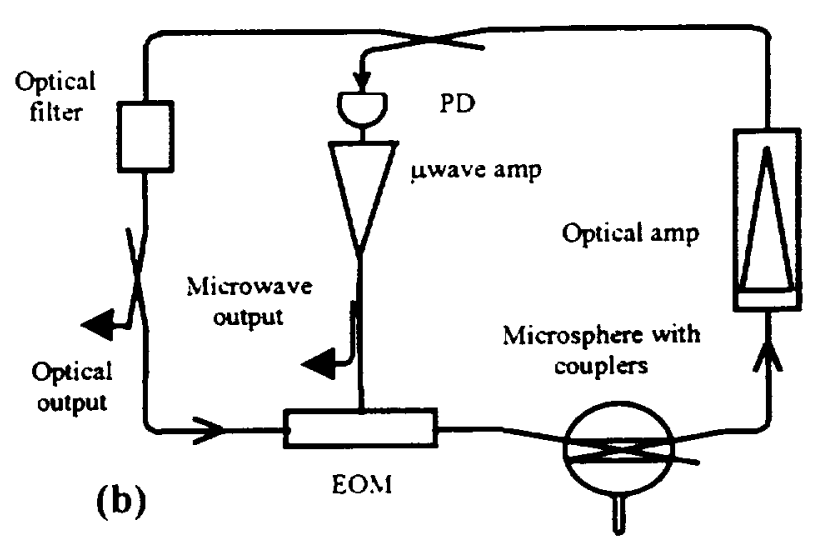

(b)

Fig.6. Schematic of microsphere-based optoelectronic oscillators: a)with external pump laset ; b) with ring laser containing microsphere and optical amplifict. Not shown are prism couplers and liber collimation optics.

With external pump laser set on the frequency of one of WG modes, the microsphere acts as a high-selectivity sideband filter. Oscillation is obtained if the gain of the microwave amplifier is higher than the total loss in the rest of the loop including the elcctro-optical modulator. input and ouput optical couplers to the sphere. and the photodetector. In the ring-laser configuration. optical oscillation is set autonatically at the frequency (frequencies) of WG modes, and the scheme works as a variety of earlier-reported coupled optoelectronic microwave oscillator. COEO [28]. We have tested the latter configuration in (wo variants: a)with fiber-pigtailed seiniconductor optical amplificr and b) with crbium-doped fiber amplifier. In all these configurations. we inanaged to readily obtain oscillation at few integral inultiples $(n=1-3)$ of the "small" free spectral range of microsphere ( $-6 \mathrm{GH} z$ in our experiment), limited by the response of the photodector in use. 
Results of preliminary experiment are illustrated in Fig.7. Fig.7a presents the optical spectrum of the microsplere-based ring laser including the semiconductor optical amplifier (E-Tek LDOA-1300). With the microwave loop open and no optical filter. the spectrum contains several components separated by $0.98 \mathrm{~nm}$ - "large" free spectral range of the sphere. They correspond to successive principal mode index values $/$ in the vicinity of 1,250 : sphere diameter in the experiment was $\sim 360 \mu \mathrm{m}$. With the optical filter in the loop, only one of the "large" FSR spectrum component survives, and stable microwave oscillation can be obtained in the complete microwave loop of COEO.

Presented in Fig. $7 \mathrm{~b}$ is a typical spectrum of microwave oscillation in a complete COEO with erbium fiber-optic amplifier (Nortel FA-17) and tunable fiber-optic filter (DiCon TE-9-1565).
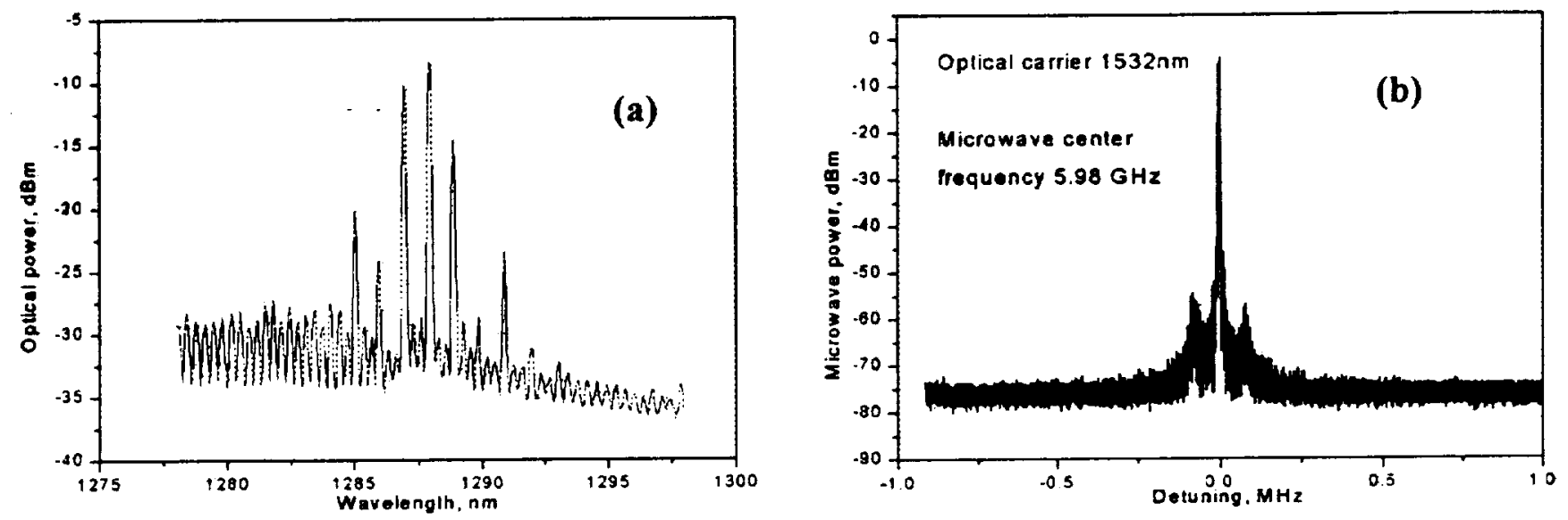

Fig.7. Optical spectrum of the microsphere based ring laser including semiconductor optical amplifier (a) and microwave oscillation spectrum of the erbium-amplifier based COEO (b). Fused silica microsphere $360 \mu \mathrm{m}$ in diameter, "small" free spectral range $5.98 \mathrm{GHz}$; unloaded quality-factor $\mathrm{Q}=1.2 \times 10^{8}$

\section{CONCLUSION}

High-Q microsphere cavity with whispering-gallery modes is a promising new component for fiber optics and photonics applications. Experiments on diode laser stabilization using optical feedback from microspheres, and optoelectronic oscillation based on microwave sideband selection by whispering-gallery modes, demonstrate their potential for the realization of ultra-compact narrow-line oscillators in optical and microwave band. The ongoing development of compact and efficient coupling techniques, inluding the reported simple fiber-optic coupler, will facilitate their incorporation with existing fiber optics and planar integrated optics devices.

\section{ACKNOWLEDGEMENTS}

The research described in this paper was carried out by the Jet Propulsion Laboratory. California Institute of Technology, under a contract with the National Aeronautics and Space Administration.

\section{REFERENCES}

1. V.B.Braginsky. M.L.Gorodetsky. V.S.Ilchenko, "Quality-factor and nonlinear properties of optical whispering-gallery modes". Phys. Lett. A137. pp.393-6. 1989

2. S.Schiller. R.Byer. "High-resolution spectroscopy of whispering-gallery modes in large dielectric spheres". Opt.Lett., 16. pp. 1138-40. 1991.

3. L.Collot. V.Lefevre-Seguin, M.Brune. J.-M.Raimond. S. Haroche, "Very high-Q whispering-gallery mode resonances observed on fused silicil microspheres". Europhys. Lett. 23(5), pp.327-33. 1993. 
4. H.Mabuchi. H.J.Kimble. "Atom galleries for whispering atoms: binding atoms in stable orbits around a dielectric Calvity". Opt.Lett. 19, pp.749-51, 1994

5. D.J.Norris. M.Kuwata-Gonokami. W.E.Moemer. "Excitation of a single molecule on the surface of a spherical microcavity", Appl.Phys.Lett., 71. pp. 297-9. 1997

6. D.W.Vemooy, A.Furusawa. N.P. Georgiades. V.S.Ilchenko. H.J.Kimble. "Cavity QED with high-Q whispering-gallery modes", Phys.Rev., A57, pp.R2293-6, 1998

7. M.Nagai. F.Hoshino, S. Yamamoto, R.Shimano, M.Kuwata-Gonokami. "Spherical cavity-mode laser with self-organized $\mathrm{CuCl}$ microspheres", Opt.Lett., 22, pp. 1630-32, 1997

8. V.Sandoghdar, F.Treussart. J.Hare. V.Lefêvre-Seguin, J.-M.Raimond. S.Haroche.. "A very low threshold whisperinggallery mode microsphere laser", Phys. Rev.. B54, pp.R1777-80, 1996

9. F.Treussart, V.S.Ilchenko, J.-F.Roch, P.Domokos, J.Hare. V.Lefevre-Seguin, J.-M.Raimond, S.Haroche, "Whisperinggallery mode microlaser at liquid-helium temperature", J.Lumin., 76, pp.670-73, 1998

10. M.L.Gorodetsky, S.P.Vyatchanin, V.S. Ilchenko, "Coupling and tunability of optical whispering-gallery modes: A basis for coordinate meter", Opt.Commun., 107, pp. $+1-8,1994$

11. A.Serpengüzel, S.Amold, G.Griffel, "Excitation of resonances in microspheres on an optical fiber", Opt.Lett., 20, pp.654-6, 1995.

12. R.K.Chang, A.J.Campillo, eds., Optical processes in microcavities, World Scientific, Singapore, 1996

13. M.L.Gorodetsky, A.A.Savchenkov, V.S.Ilchenko, "Ultimate $Q$ of optical microsphere resonators", Opt.Lett., 21, pp.4535,1996

14. D.W.Vernooy, V.S.Ilchenko, H.Mabuchi, E.W.Streed, H.J.Kimble, "High-Q measurements of fused-silica microspheres in the near infrared", Opt.Lett., 23, 247-9, 1998

15. V.V.Vasilev, V.L. Velichansky, M.L.Gorodetcky, V.S.Ilchenko, L.Hollberg, A.V.Yarovitsky, "High-coherence diode laser with optical feedback via a microcavity with whispering-gallery modes", Quantum Electronics, 23(8), pp.675-6, 1996.

16. X.S.Yao, L.Maleki, "Converting light into spectrally pure microwave oscillation". Opt.Lett., 21, pp.483-5, 1996.

17. S. Todoroki, S. Sakaguchi, M. Peeters, "Refractive index dispersion of sodium magnesium silicate glasses for ultralowloss fibers", J. Amer. Ceramic Soc., 80, pp.313-6, 1997.

18. B.E.Little, H.A.Haus, E.P.Ippen, E.R.Thoen, G.Steinmeyer, J.S. Foresi, L.C.Kimerling, S.T.Chu, W.Greene, "Microresonators for integrated optical devices", Opt. \& Photonics News, 9, pp. 32-3, 1998.

19. R.D.Richtmyer, "Dielectric resonators", JAppl.Phys., 10, pp.391-8, 1939.

20. G.Chen, R.K.Chang, S.C.Hill, P.W.Barber, "Frequency splitting of degenerate spherical cavity modes: stimulated Raman scattering spectrum in deformed droplets", Opt.Lett., 16, pp.1269-71, 1991.

21. M.L.Gorodetsky and V.S.Ilchenko, "High-Q whispering-gallery microresonators: Precession approach to spherical mode analysis and emission patterns with prism couplers", Opt.Commun., 113, pp.133-43, 1994

22. V. S. Ilchenko, P. S. Volikov, V.L. Velichansky, F. Treussart, V. Lefevre-Seguin, J.-M. Raimond, S.Haroche, "Straintunable high-Q optical microsphere resonator", Opt.Commun., 145, pp.86-90, 1998.

23. N.Dubreuil, J.C.Knight, D.Leventhal, V.Sandoghdar, J.Hare, and V.Lefevre-Seguin, J.-M.Raimond, S.Haroche, "Eroded monomode optical fiber for whispering-gallery mode excitation in fused-silica microspheres", Opt.Lett., 20, 1515-8, 1995.

24. J.C.Knight, G.Cheung, F.Jacques, T.A.Birks, "Phase-matched excitation of whispering gallery mode resonances using a fiber taper", Opt.Lett., 22, pp.1129-1131, 1997.

25. M.L.Gorodetsky. V.S.Ilchenko, "Optical microsphere resonators: optimal coupling to high-Q whispering-gallery modes", J.Opt. Soc.tmerica, B16. pp.147-54, 1999.

26. C.C.Lam. P.T.Leung. K.Young. "Explicit asympthotic formulas for the positions. widths, and suregths of resonances in Mie scattering", J.Opt.Soc.America, B9. pp.1585, 1992.

27. V.V.Vassiliev. V.L. Velichansky. V.S.Ilchenko. M.L.Gorodetsky. L. Hollberg. A.V.Yarovitsky, "Narroiv-linewidth diode laser with a high-Q microsphere resonator", accepted for publication in Opt.Commun., 1999.

28. X.S.Yao. L.Maleki. "Dual microwave and optical oscillator". Opt.Lett., 22. pp. 1867-9. 1997 